\title{
Examining the Role of HIPPO/YAP and AMOT Dysregulation and Imbalance in Autosomal Dominant Polycystic Kidney Disease John Underwood ${ }^{1}$, Robert Bacallao ${ }^{1}$ \\ 1 Indiana University School of Medicine, Department of Nephrology
}

Autosomal Dominant Polycystic Kidney Disease (ADPKD) is a genetic disorder, most commonly caused by mutations in Polycystin-1 and Polycystin-2 (PC1 and PC2), in which many large lumenal cysts develop in the kidney epithelium. The disease is progressive and ultimately leads to renal dialysis and/or kidney transplant given the lack of efficacious therapeutic intervention. Current research has implicated HIPPO/YAP pathway dysregulation and AMOT imbalance following defects in cell-cell adhesion and contact inhibition as the molecular basis for ADPKD. To test this hypothesis as to the minimum cellular changes necessary to produce cystogenesis, the Bacallao lab microinjected normal human kidney epithelial (HK2) cells in order to drive ectopic expression of Cadherin-8 (CAD-8). Our Imaging with confocal microscopy and subsequent image analysis indicates CAD-8, a cadherin abnormally expressed in renal epithelial cells of ADPKD patients, is sufficient to cause formation of cysts albeit with uncharacteristically small lumens compared to typical ADPKD cysts. However, these cells exhibit significantly larger cyst expansion when they are transduced for coexpression of CAD-8 and constitutively active YAP5SA. Cells modified for heterozygosity with respect to a defective PC1 gene (PKD) also exhibited significantly larger lumen cysts when transduced with YAP5SA reinforcing the role of Yap in lumen size control. Finally, PKD cells transduced with AMOTL1 exhibited significantly smaller lumen sizes. AMOTL 1 belongs to a family of adapter proteins which bind and inhibit Yap activity thus showing conclusively that positive and negative modulation of Yap activity correlates with cyst lumen size expansion and reduction respectively.

“This project was funded, in part, with support from the O'Brien Center for Advanced Renal Microscopic Analysis funded, in part by P30 DK079312 from the National Institutes of Health. The content is solely the responsibility of the authors and does not necessarily represent the official views of the National Institutes of Health." 